\title{
Comunicación
}

\section{Intoxicación natural de avestruces por consumo excesivo de frutos de Samanea saman en Colombia}

\author{
Natural poisoning of ostriches due to excessive consumption of Samanea saman \\ fruits in Colombia
}

Walter Hernández-Arroyave ${ }^{1,3}$, John Jairo Bustamante-Cano²

\section{Resumen}

\begin{abstract}
Se expone un cuadro de intoxicación natural de dos ejemplares de avestruz (Struthio camelus), criados en cautiverio, a consecuencia del consumo excesivo de frutos de samán (Samanea saman) en la región del Magdalena Medio Colombiano. Las aves presentaban mal estado corporal, alto grado de deshidratación, comportamiento apático, debilidad, somnolencia y posteriormente su muerte. En la necropsia, los hallazgos macroscópicos más sobresalientes fueron localizados en el sistema gastrointestinal, con grave impactación del proventrículo a causa del acúmulo de frutos y semillas de la leguminosa y una extensa área de mucosa ulcerada. El riñón fue el órgano con mayores lesiones histopatológicas típicas de un cuadro nefrotóxico. No hubo lesiones en hígado. Las transaminasas glutámico oxalacética (TGO) y glutámico pirúvica (TGP) se encontraron elevadas. Pruebas colorimétricas revelaron la presencia de taninos y saponinas del material vegetal hallado en proventrículo. Los resultados indicaron que los avestruces murieron a causa de intoxicación por consumo de frutos del samán.
\end{abstract}

Palabras clave: avestruz; intoxicación: nefrotoxicidad; samán

\footnotetext{
${ }^{1}$ Grupo de Investigación en Producción y Ciencia Animal (PROCA), Escuela de Medicina Veterinaria y Zootecnia, Universidad de la Paz - UNIPAZ, Barrancabermeja, Santander, Colombia

${ }^{2}$ Departamento de Medicina Veterinaria, Facultad de Ciencias Agrarias, Universidad de Pamplona, Pamplona, Colombia

${ }^{3}$ E-mail: walter.arroyave@unipaz.edu.co
}

Recibido: 14 de febrero de 2019

Aceptado para publicación: 27 de octubre de 2019 
A natural intoxication of two specimens of ostrich (Struthio camelus), raised in captivity, as a result of excessive consumption of fruits of saman (Samanea saman) in the Magdalena region of Colombia is presented. The birds had poor body condition, high degree of dehydration, apathy, weakness, drowsiness and subsequently death. The most outstanding macroscopic findings during the necropsy was a severe impaction of the proventriculus due to the accumulation of fruits and seeds of the legume and an extensive area of ulcerated mucosa. The kidney was the organ with the greatest histopathological lesions typical of a nephrotoxic condition. There were no liver lesions. Glutamic oxalacetic (TGO) and glutamic pyruvic (TGP) transaminases were found elevated. Qualitative colorimetric tests revealed the presence of tannins and saponins of the plant material found in proventriculus. The results indicated that the ostriches died due to poisoning due to the consumption of fruits of the saman.

Key words: ostriches; intoxication; nephrotoxicity; saman

\section{INTRODUCCIÓN}

El empleo de leguminosas en sistemas para la alimentación animal es un ejemplo típico de las técnicas alternativas que han sido resaltadas por varios autores, quienes defienden su utilización por tener un elevado valor alimenticio y ser ricas en proteínas, minerales y otros principios nutritivos (García y Medina, 2006). Sin embargo, es importante tener en cuenta la presencia de metabolitos secundarios tóxicos en estas plantas (Jackson et al., 1996). Algunos de estos compuestos solo se encuentran en determinadas especies y cumplen funciones ecológicas específicas o como mecanismos naturales de defensa contra herbívoros o microorganismos patógenos (García y Medina, 2006).

Samanea saman (samán, algarrobillo, campano) es una fabácea de gran tamaño, oriundo del trópico seco americano, que se ha generalizado en todo el trópico húmedo y subhúmedo. Es un árbol que produce gran cantidad de frutos (vainas) de alta calidad nutritiva constituyéndose en un importante suplemento forrajero para el ganado durante el periodo poco lluvioso (Roncallo, 2009); sin embargo, contiene altas concentraciones de metabolitos secundarios con potenciales efectos tóxicos como polifenoles (taninos) glucósidos (saponinas) y otros terpenoides (Harley, 2017). En Colombia se han descrito algunos efectos tóxicos en ganado bovino producidos por el consumo de frutos de Enterolobium cyclocarpum, otra leguminosa de la familia fabácea, pero no se encuentran reportes bibliográficos sobre los efectos tóxicos en avestruces por consumo de frutos de $S$. saman.

El presente trabajo describe la intoxicación de avestruces, de manera natural, debido al consumo excesivo del fruto de Samanea saman en un criadero ubicado en la región del Magdalena Medio, Colombia.

\section{Caso Clínico}

El caso se presentó en un criadero de avestruces localizado en el municipio de La Mata, departamento del Cesar, región del Magdalena Medio, Colombia. La zona se encuentra a $200 \mathrm{~m}$ sobre el nivel del mar y es considerada como bosque muy seco tropical 
(bms-T), según la clasificación de Holdridge. El rebaño estaba conformado por 27 avestruces Struthio camelus de ambos sexos y diferentes edades. Los animales eran mantenidos bajo condiciones de manejo semi-intensivo, con pasturas naturales, y disponiendo de buen sombrío de samán y de guácimo (Guazuma ulmifolia). Los adultos eran alojados en tríos (dos hembras y un macho) en corrales de $1200 \mathrm{~m}^{2}$, disponiendo, además, de un amplio corredor para ejercicio y de cobertizos con abundante agua, y donde se les ofrecía una pequeña ración de concentrado, cada dos o tres veces a la semana.

El motivo de la consulta fue el estado general de dos avestruces, con pérdida de peso, plumas sin lustre y comportamiento apático. Durante la visita al establecimiento se constató que los dos animales afectados correspondían a una hembra y a un macho adultos que hacían parte de un trío, y cuyo tercer animal había fallecido meses atrás por causas no definidas. Los animales afectados presentaban signos clínicos muy idénticos que incluían apatía, debilidad, respiración agitada, pobre estado corporal, plumas erizadas y sin lustre, deshidratación, timpanismo y heces secas recubiertas de moco y sangre. Además, era notorio el prolapso del órgano copulador en el macho.

Se tomaron muestras de sangre para estudios de química sanguínea. Unas horas después de la evaluación clínica, los avestruces entraron en recumbencia lateral, depresión, somnolencia y muerte (Figura 1). Inmediatamente después de la muerte se procedió con la necropsia, remitiendo cortes de piezas en alcohol tamponado al laboratorio para estudio histopatológico.

En el examen post mortem se determinó que la condición corporal de ambos animales era regular. No se hallaron depósitos de grasa ni se evidenció la presencia de ectoparásitos. En la inspección de la región abdominal fueron observadas, in situ, todas las estructuras internas. Luego, fueron extraídas las asas intestinales, en las cuales se

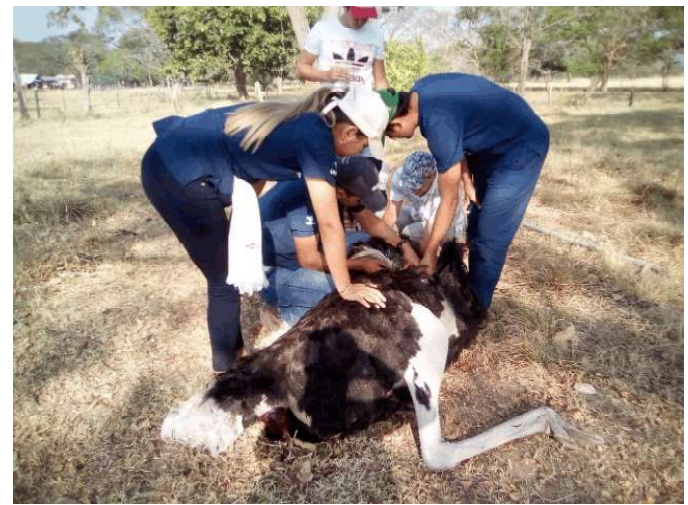

Figura 1. Evaluación de uno de los dos avestruces en recumbencia

visualizaron ulceraciones focales en numerosos puntos del trayecto de intestino delgado (Figura 2). La molleja y el proventrículo presentaban gran compactación, producto de la gran acumulación de material vegetal, que en su mayoría resultó ser frutos y semillas de samán. Algunos hallazgos en la molleja fueron apreciables, como por ejemplo, el color marrón de la capa superficial, la presencia de úlceras, el adelgazamiento de la capa de queratina, su facilidad para desprenderse y la gran fragilidad al tacto. Así mismo, el riñón se mostraba pálido, levemente engrosado, sin que se denote la delimitación de los lóbulos craneal y medial en los dos casos. Los demás órganos no presentaban alteraciones macroscópicas de relevancia.

En el análisis histopatológico se encontraron daños en el tubo digestivo correspondientes a congestión severa y edema moderado, con infiltrado inflamatorio mixto en la mucosa de extensas áreas de proventrículo, yeyuno e íleon; mientras que en la submucosa se observó edema moderado y congestión generalizada. En el hígado, en términos generales, se encontró predominio de alteraciones microcirculatorias moderadas, cambios vacuolares leves y algunos focos de degeneración hidrópica. Por otra parte, el tejido renal presentó cambios microscópicos de relevancia que correspondieron a lesiones típicas de un cuadro nefrotóxico severo de tipo 


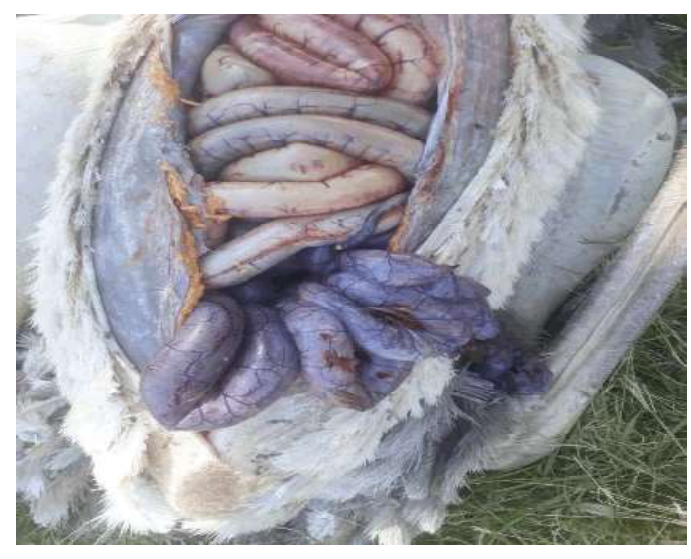

Figura 2. Congestión y ulceraciones en intestino delgado de uno de los dos avestruces afectados.

agudo, que fueron caracterizadas por necrosis de células epiteliales de grandes segmentos de túbulos contorneados proximales, edema y fibrosis intersticial; mientras que en los túbulos distales se observó epitelio aplanado con dilatación de su luz.

El perfil enzimático de los avestruces afectados se muestra en el Cuadro 1. Fragmentos del material vegetal hallados en molleja y proventrículo de los dos avestruces fueron analizados para su identificación botánica en el herbolario de la Universidad de Pamplona (Norte de Santander, Colombia), determinándose que se trataba de S. saman. En la identificación de metabolitos secundarios, como taninos y saponinas, fueron utilizadas pruebas cualitativas (colorimétricas). Para los primeros se utilizaron las reacciones con tricloruro de hierro y ácido fosfowolfrámico, y para el segundo grupo se utilizó la Prueba de Liebermann-Burchard, dando resultados positivos en ambos casos.

\section{Discusión}

Los frutos de $S$. saman, además de su alto valor proteico, poseen carbohidratos y saponinas. Estas últimas pertenecen al grupo de triterpenos y su actividad está relacionada con la disminución de la tensión superficial de los líquidos dentro del ambiente ruminal, produciendo espuma, lo cual genera las condiciones propicias para la acumulación de gas que conlleva a la formación del timpanismo espumoso (Wang et al., 2012).

En un estudio acerca del tamizaje fitoquímico de las vainas del samán se reveló la presencia de moderadas cantidades de los principales metabolitos secundarios, entre ellos flavonoides, resinas, glucósidos triterpenoides (saponinas), y polifenoles (taninos); mientras otros estudios demostraron que los frutos de $S$. saman pueden contener hasta $45 \mathrm{mg} / \mathrm{g}$ de taninos condensados extractables (Hess et al., 2003; Obasi et al., 2010).

Un análisis cualitativo de estos taninos condensados (catecoles) mostró que están formados por cianidina, catequina, epicaquina, antocianidina monoglicones, delphinidina y malidina (Ukoha et al., 2011), los cuales están presentes en los frutos carnosos del $S$. saman $\mathrm{y}$, posiblemente, sean los que induzcan los estados morbosos en el organismo animal (Soica et al., 2010; Costa et al., 2011; Wang et al., 2012). Si se consideran las características bromatológicas de estas semillas, ricas en carbohidratos de fácil fermentación, así como la alta ingestión por parte de los animales, pueden ser razones que provoquen una serie de condiciones patológicas que en muchos casos llevan a un desenlace fatal (Sánchez-Bell, 2015).

Las manifestaciones clínicas observadas en los avestruces afectados podrían estar relacionadas con el consumo excesivo de los frutos de samán. Se ha observado que las manifestaciones clínicas en el ganado bovino y ovino son de carácter estacional, durante la etapa final del período seco, coincidiendo con el escaso rendimiento de los pastos, donde las manifestaciones morbosas comienzan entre 15 y 25 días de haber comenzado la ingestión de las semillas a voluntad (SánchezBell, 2015). 
Cuadro 1. Perfil de enzimas séricas en dos avestruces (Struthio camelus) intoxicados por consumo de samán

\begin{tabular}{lccc}
\hline Enzimas & Avestruz 1 & Avestruz 2 & $\begin{array}{c}\text { Valores } \\
\text { normales }^{1}\end{array}$ \\
\hline Aspartato aminotransferasa - TGO (UI/1 & 237 & 330 & 190 \\
Alanino aminotransferasa - TGP (UI/l) & 16.9 & 16.1 & 20.62 \\
Creatin kinasa - CK (UI/l) & 1650 & 1661 & 1655 \\
Creatinina (mg/dl) & 0.45 & 0.48 & 0.22 \\
Nitrógeno ureico en sangre - BUN (mg/dl) & 6.75 & 6.5 & 3.2 \\
Albúmina (g/dl) & 1.18 & 1.21 & 1.77 \\
Proténas totales - PT (g/l) & 24.7 & 32.2 & 46 \\
\hline
\end{tabular}

${ }^{1}$ Fuente: Palomeque et al. (1991)

El avestruz, herbívoro por naturaleza, también se alimenta de plantas suculentas, semillas, bayas, hierbas y hojas de árboles y arbustos en su entorno natural. Su colon y ciego de gran tamaño sirve como cámara de fermentación que produce altas concentraciones de ácidos grasos volátiles y contiene bacterias que digieren la semicelulosa y la celulosa (Dabrowskis, 2002; Aganga et al., 2003), lo que lo asemeja a la fisiología digestiva del caballo (Swart et al., 1993). Por otro lado, el timpanismo observado en los dos avestruces del presente caso podría relacionarse con factores tales como el alto contenido de proteínas, la alta digestibilidad de las vainas de saman y el alto contenido de saponinas que favorecen la producción de espumas (Hess et al., 2003; Wang et al., 2012).

Roa (2016) evaluó en los Llanos Orientales de Colombia los efectos tóxicos del Enterolobium cyclocarpum, de la misma familia de las fabáceas, encontrando un incremento en la enzima aspartato transaminasa (TGO) en los bovinos intoxicados experimentalmente. TGO es un indicador de lesión hepática, músculo, encéfalo, riñón y células sanguíneas (Villa et al., 1999).

\section{Conclusiones}

Los hallazgos encontrados en este caso clínico presuponen el efecto nocivo en avestruces (Struthio camelus) de ciertos metabolitos secundarios presentes en Samanea saman.

\section{Literatura Citada}

1. Aganga AA, Aganga AO, Omphile UJ. 2003. Ostrich feeding and nutrition. Pak J Nutr 2: 60-67.

2. de Costa F, Yendo AC, Fleck JD, Gosmann G, Fett-Neto AG. 2011. Immunoadjuvant and anti-inflammatory plant saponins: characteristics and biotechnological approaches towards sustainable production. Mini Rev Med Chem 11: 857-880. doi: 10.2174/ 138955711796575470

3. Dabrowskis G. 2002. Alimentación y nutrición de avestruces. En: Memorias XI Congreso Venezolano de Producción e Industria Animal. Valera, Venezuela. 
4. García D, Medina M. 2006. Composición química, metabolitos secundarios y aceptabilidad relativa de diez árboles forrajeros. Zootec Trop 24: 233-250.

5. Naumann HD, Tedeschi LO, Zeller WE, Huntley NF. 2017. The role of condensed tannins in ruminant animal production: advances, limitations and future directions. Rev Bras Zootecn 46: 929-949. doi: 10.1590/s1806-92902017001200009

6. Hess HD, Kreuzer M, Diaz TE, Lascano CE, Carulla JE, Soliva CR, Machmuller A. 2003. Saponin rich tropical fruits affect fermentation and methanogenesis in faunated and defaunated rumen fluid. Anim Feed Sci Tech 109: 79-94. doi: 10.1016/S03778401(03)00212-8

7. Jackson F, Barry T, Lascano C, Palmer B 1996. The extractable and bound condensed tannin content of leaves for tropical tree, shrub and forage legumes. J Sci Food Agr 17: 103-110. doi: 10.1002/(SICI)1097-0010-(199605)71:1<103::AID-JSFA554>-3.0.CO;2-8

8. Obasi NL, Egbuonu AC, Ukoha PO, Ejikeme PM. 2010. Comparative phytochemical and antimicrobial screening of some solvent extracts of Samanea saman (Fabaceae or mimosaceae) pods. Afr J Pure Appl Chem 4: 206-212.

9. Palomeque J, Pinto D, Viscor G 1991. Hematologic and blood chemistry values of the Masai ostrich (Struthio camelus). J Wildl Dis 27: 34-40. doi: 10.7589/00903558-27.1.34

10. Roa L. 2016. Caracterización clínica y anatomopatológica de la intoxicación por Enterolobium cyclocarpum en el gana- do bovino en los Llanos Orientales de Colombia. Tesis de Maestría. Bogotá, Colombia: Univ. Nacional de Colombia. 62 p.

11. Roncallo B, Torres E, Sierra M. 2009. Producción de vacas de doble propósito suplementadas con frutos de algarrobillo (Pithecellobium saman) durante las 1luvias. [Internet]. Disponible en: http:// www.fao.org/3/Y4435S/y4435s0m.htm

12. Sánchez-Bell W. 2015. Manejo sanitario del algarrobo (Pithecellobium saman). Rev Granma Ciencia 19(1).

13. Soica C, Peevm C, Cirulea R, Dehelean C. 2010. Physico-chemical and toxicological evaluations of betulin and betulinic acid interactions with hydrophilic cyclodextrins. Farmacia 58: 611-619.

14. Swart D, Mackie R, Hayes JP. 1993. Influence of live mass, rate of passage and site of digestion on energy metabolism and fibre digestion in the ostrich (Struthio camelus var. domesticus). S Afr J Anim Sci 23: 119-126.

15. Ukoha PO, Cemaluk EAC, Nnamdi OL, Mad PE. 2011. Tannins and other phytochemical of the Samanaea saman pods and their antimicrobial activities. Afr J Pure Appl Chem 5: 237-244.

16. Villa NA, Ceballos A, Cerón D, Serna CA. 1999. Valores bioquímicos sanguíneos en hembras Brahman bajo condiciones de pastoreo. Pesqui Agropecu Bras 34 :2339-2343. doi: 10.1590/S0100204X1999001200021

17. Wang Y, Majak W, McAllister T. 2012. Frothy bloat in ruminants: cause, occurrence, and mitigation strategies. Anim Feed Sci Tech 172: 103-114. doi: 10.1016/j.anifeedsci.2011.12.012 